Detschew, Vesselin; Funkat, Gert; Kaeding, Anne-Kathrin:

Moderne informationstechnologische Werkzeuge bei der Realisierung von medizinischen Leitlinien-Servern

Zuerst erschienen in: Biomedizinische Technik = Biomedical Engineering. Berlin [u.a.] : de Gruyter. - 45 (2000), S1, S. 351-352.

Erstveröffentlichung: 2000

Datum Digitalisierung: 2009-07-17

ISSN (online): $\quad$ 1862-278X

ISSN (print): $\quad$ 0013-5585

DOI: $\quad$ 10.1515/bmte.2000.45.s1.351

[Zuletzt gesehen: 2019-08-20]

„Im Rahmen der hochschulweiten Open-Access-Strategie für die Zweitveröffentlichung identifiziert durch die Universitätsbibliothek IImenau. "

"Within the academic Open Access Strategy identified for deposition by IImenau University Library."

„Dieser Beitrag ist mit Zustimmung des Rechteinhabers aufgrund einer (DFG-geförderten) Allianz- bzw. Nationallizenz frei zugänglich."

"This publication is with permission of the rights owner freely accessible due to an Alliance licence and a national licence (funded by the DFG, German Research Foundation) respectively."

\section{DFG}

Nationallizenzen 


\title{
MODERNE INFORMATIONSTECHNOLOGISCHE WERKZEUGE BEI DER REALISIERUNG VON MEDIZINISCHEN LEITLINIEN-SERVERN
}

\author{
V. Detschew, G. Funkat, A.-K. Kaeding \\ Fachgebiet Medizinische Informatik \\ Technische Universität Ilmenau, Deutschaland \\ vde@informatik.tu-ilmenau.de
}

\section{EINLEITUNG}

Leitlinien sind systematisch entwickelte Darstellungen und Empfehlungen mit dem Zweck, Ärzte und Patienten bei der Entscheidung über zweckdienliche Maßnahmen der Krankenversorgung (Prävention, Diagnostik, Therapie und Nachsorge) unter spezifischen klinischen Umständen zu unterstützen.

In Deutschland gibt es über 550 von Fachgesellschaften entwickelte Leitlinien. Sie integrieren unter Zuhilfenahme des Instruments der Evidence Based Medicine (EBM) wissenschaftliche Erkenntnisse im Hinblick auf eine konkrete Problemstellung und formulieren eine standardisierte Abfolge sinnvoller Schritte sowie zulässige Abweichungen vom sog. Behandlungskorridor. Sie sollen den neuen Erkenntnissen und der modernen Entwicklung flexibel und rasch angepasst werden. Sie sollen vor allem der Verbesserung der Qualität und der Steigerung der Effizienz im Bereich der Gesundheitsversorgung dienen.

\section{BESTEHENDE ANSÄTZE}

Wie entstehen Leitlinien? - Leitlinien entstehen in Konsensusprozessen.

Welche Ziele werden dabei verfolgt?

- Erhöhung der Behandlungsqualität

- Vorhersagbarkeit der Patientenbehandlung

- Steigerung der Patientenzufriedenheit

- Steigerung der Sicherheit beim Personal

- Möglichkeit der Kostenkontrolle

- Verbesserung der Wettbewerbssituation

Welche technischen Lösungen sind heute möglich?

- Leitlinie auf Papier - Die Leitlinie auf dem Papier ist noch die gängigste Lösung. Die häufigste Form ist eine Leitlinie als Fließtext, seltener in Form von Diagrammen oder Entscheidungsbäumen. Nachteile: Die Einhaltung solcher Leitlinien ist schwer kontrollierbar, eine auch nur partielle Änderung von Teilschritten führt zum Austausch des gesamten Dokuments.

- Leitlinie auf einem Server im Internet - Das elektronische Abbild von papiergestützten Leitlinien ist im Internet bei den Fachgesellschaften einzusehen, wo die Leitlinien als HTML-Dokument vorliegen [2]. Die technischen Lösungen für den Zugriff bestehen im allgemeinen aus einzelnen PC's mit
HTML-Browser, über die im Internet oder im klinikinternen Intranet in relevanten Leitlinien gesucht wird (Leitlinienserver). Die HTML-Dokumente stellen das Wissen nicht strukturiert dar. In den meisten Fällen kann diese Leitlinie bei Bedarf angepasst oder geändert werden. Hinweise über solche Änderungen können auch auf elektronischem Wege, z.B. per E-Mail erfolgen.

- Leitlinienserver als Nachschlagewerk - Eine Erweiterung der Funktionalität ist die Ergänzung der standardisierten Leitlinien durch Verweise auf andere Quellen medizinischen Wissens in Form von Links auf anderen Servern. Dadurch können Abweichungen in der Behandlungsabläufen validiert werden, das notwendige medizinische Wissen wird durch die jeweiligen Anbieter aktualisiert.

- Leitlinienserver als integraler Bestand von StationsInformationssystemen (SIS) - Eine Integration von Leitlinienservern in ein SIS ermöglicht die Kontrolle ausgewählter Parameter und somit die Beobachtbarkeit und Kontrolle des Behandlungsablaufes. Dabei kann der Leitlinienserver als ,,stiller Beobachter" lediglich für die Einhaltung von Behandlungsmaßnahmen sowie von Toleranzbereichen bestimmter Messwerte fungieren und diese automatisch protokollieren. Eine entsprechende Gestaltung der Software kann dabei die Kontrolle von Qualitätsparameter realisieren und somit QS - Maßnahmen effektiv unterstützen.

- Leitlinienserver als Komponente eines wissensbasierten Systems - eine Unterstützung des ärztlichen Handelns durch automatisch generierte Hinweise oder durch Auslösen von Warnungen bei kritischen Zuständen stellt die höchste mögliche Stufe der Funktionalität von elektronischen Leitlinienservern dar. Voraussetzung dafür ist eine entsprechend gestaltetet Wissensbasis für die jeweilige Problemdomäne sowie die Realisierung von wissensverarbeitenden Komponenten der Software. Notwendig ist außerdem neben der Umsetzung der Leitlinie als Wissenskomponente des Systems auch eine validierte Beschreibung des Behandlungsprozesses. 


\section{ANFORDERUNGEN AN EIN WEITERFÜHRENDES KONZEPT}

Um Leitlinien in die Praxis- und Krankenhausroutine zu implementieren ist ein mehrstufiger Prozess notwendig, der einen abgeschlossenen Konsensusprozess der Fachgesellschaften voraussetzt:

- Konsolidierte Leitlinien müssen dem Kreis der potentiellen Anwender bekannt gemacht werden und in der Handhabung erläutert werden.

- Die Zeitspanne für die Verfügbarmachung von Leitlinien muss deutlich verkürzt werden. Dazu eignen sich die aktuell verfuggbaren elektronischen Mittel.

- Durch die Erweiterung der Funktionalität können Leitlinien intelligenter gestaltet werden. Das ist in erster Linie durch wissensverarbeitende Systeme zu leisten.

Mit dem Einsatz von IT - gestützten Leitliniensystemen ergeben sich eine Reihe von weiteren Aspekten, die in der klinischen Routine zunehmend an Bedeutung gewinnen:

- Die Kontrolle der Einhaltung von Leitlinien ermöglicht die rechtliche Absicherung der medizinischen Versorgung.

- Die durch Leitlinien vorgegebenen Behandlungskorridore erlauben eine genauere Kalkulation und Kontrolle der zu erwartenden Kosten.

- Für das Management klinisch - medizinischer Versorgungsprozesse wird ein neue Stufe der Qualitätssicherung und des Qualitätsmanagement möglich.

Weitere Punkte, die durch IT - gestützte Leitlinienserver überhaupt erst ermöglicht werden:

- Automatisierte Kontrolle und Protokollierung der Behandlung

- Generierung von Empfehlungen, Führung durch die Behandlung

- Ergebnisbewertung (nicht nur kostenmäßig)

- Semantische Analyse des Behandlungsprozesses, Schwachstellenanalyse

- IT - gestütztes Qualitätsmanagement

Um Ärzte bei der Nutzung elektronischer Leitlinien zu unterstützen, muss der Zusatzaufwand so gering wie möglich gehalten werden und die Systeme müssen reibungslos in bestehende IV - Systeme integriert werden. Konkrete Realisierungen hängen von der jeweiligen aktuellen Situation und den Nutzeranforderungen ab. Eine besonders effektive Integration ist durch eine automatische Übernahme von Patientendaten und Messwerten aus elektronischen Patientenakten möglich. Die Integrationsschritte werden dabei durch die kontextsensitiven Aufrufe der Leitlinien, die intelligenten Informationssuchalgorithmen sowie die Erstellung von Empfehlungen unterstützt.

\section{ANWENDUNGSBEISPIEL}

In der intensivmedizinischen Versorgung existieren Problembereiche, die durch eine erstaunlich umfassende
Anzahl von zu bewertenden Risikoparametern beschrieben werden (SHT, Heparinisierung, Antibiose). Die Bewertung der Parameter erfordern einen großen Erfahrungsschatz, um aus einer intuitiven Einschätzung eine valide Bewertung abzuleiten. Eine objektive Einschätzung erscheint auf Grund der enormen Vielzahl von zu bewertenden Parametern problematisch.

Beispielgebend für den Bereich der Heparine wurde eine informationstechnisch gestützte Bewertung der entsprechenden Risikoparameter entwickelt. Ausgangspunkt ist die Erfassung des domänenspezifischen Wissens. Ziel der Erfassung des Expertenwissens ist die Beschreibung der Vorgehensweise des medizinischen Experten bei der Lösung eines konkreten Problemkomplexes, die Formalisierung dieses Wissens und letztlich die Abbildung in einem Leitlinienserver, mit dem Entscheidungen unterstützt werden können.

Diese Effizienz ist informationstechnologisch, wenn überhaupt, nur durch das Ableiten von Daten und Informationen aus einem bestehenden Krankenhausinformationssystem bzw. aus der Elektronischen Patientenakte möglich. Diese Daten und Informationen gehen dann direkt in die Risikobewertung eingehen, um eine automatisierte Verarbeitung zu ermöglichen.

\section{LITERATURHINWEISE}

[1] Gerlach, F. M. ; Beyer, M. ; Szecsenyi, M. ; Fischer, G. C.: Leitlinien in Klinik und Praxis. Dt Ärztebl 1998; 95: A-1014-1021 [Heft 17]

[2] Erarbeitung von Leitlinien für Diagnostik und Therapie: Leitlinien von Fachgesellschaften. AWMF online: $\mathrm{http} / / / \mathrm{www}$.uniduesseldorf.de/WWW/AWMF/11/11 list.htm

[3] Leitfaden für die Behandlung des NichtInsulinabhängigen Diabetes mellitus (NIDDM, Typ 2): ein Beitrag zur praktischen Umsetzung der St.Vincent-Declaration / European NIDDM Policy Group, Mainz: Kirchheim, 1994, ISBN 3-87409088-4

[4] Erarbeitung von Leitlinien fur Diagnostik und Therapie: Kriterien für die Qualität von Leitlinien. Arbeitsgemeinschaft der Medizinischen Fachgesellschaften. AWMF online: http://www.uniduesseldorf.de/WWW/AWMF/1//1 quali.htm

[5] Ärztliche Zentralstelle Qualitätssicherung (gemeinsame Einrichtung von Bundesärztekammer und Kassenärztlicher Bundesvereinigung): Checkliste "Methodische Qualität von Leitlinien". Deutsches Ärzteblatt 95, Heft 41, Seite A-2576

[6] FunKat, G., KAEDING, A.-K., DeTSChew, V.: Benefit and problems of medical guideline server. European Medical \& Biological Engineering Conference EMBEC'99, Vienna (Austria), Nov. 04 - 07, 1999

[7] KAEDING, A.-K.; FUNKAT, G. DETSChEW, V.: The use of scenarios as a basic concept in knowledge acquisition in medicine. European Medical \& Biological Engineering Conference EMBEC'99, Vienna (Austria), Nov. 04 - 07, 1999 